\title{
Responsiveness to Discovery
}

Primary author:

Marc Neveu - University of Maryland, College Park, MD / NASA Goddard Space Flight Center, Greenbelt, MD - Phone: +1 301-614-6872 - Email: marc.f.neveu@nasa.gov

Co-author:

Shannon M. MacKenzie - Johns Hopkins University Applied Physics Laboratory, Laurel, MD 


\section{Responsiveness to Discovery - Key points:}

1. We encourage the Decadal Survey panels and/or Steering Committee to discuss whether to establish a formal path by which NASA's Planetary Science Division can request the National Academies to adjust Decadal Survey priorities in response to scientific discoveries.

2. This white paper provides an initial discussion of example shifts from Vision \& Voyages Decadal Survey recommendations, pros and cons of such shifts, and possible means of handling responsiveness to discovery in implementing Decadal Survey recommendations.

3. This white paper does not address a response to politically mandated changes in priorities, which we understand takes precedence over scientifically driven changes in NASA decisions.

\section{Unexpected twists in the past decade}

The Planetary Budget: A rough start, a strong middle, rough finish ahead? This decade started and is ending with an economic crisis that resulted in decreased budgets for planetary science at the beginning of the decade and may (or may not, depending in part on the nature of government stimuli) do so again in the coming years. In between, planetary science was funded above the forecasts of the Vision \& Voyages Decadal Survey, in large part due to strong bipartisan support in Congress. This allowed planetary science to remain healthy by supporting the new start of two Flagship, one New Frontiers, presumably five (and at least three) Discovery, two planetary defense, and several smaller missions and contributions to international efforts.

Shifting priorities. For the most part, V\&V priorities were followed despite the above budgetary swings. Notably however, below are four examples of deviation from the priorities set in V\&V. Each of these received both pushback as not being compliant with Decadal priorities set in Vision \& Voyages, and support as either following the spirit (if not the letter) of V\&V or otherwise being scientifically worthy of undertaking.

Europa Lander. Following a political push from Europa enthusiast Rep. Culberson, then chairman of the committee in charge of approving NASA's budget, work on a Europa Lander received a large, dedicated (earmarked) budget that grew the planetary science budget envelope. Although the lack of reelection of this representative has jeopardized the implementation of this mission as originally conceived, the budget spent on Europa Lander studies and technology development can be readily leveraged for future lander concepts on essentially any outer solar system moon, and to a lesser extent at other planetary bodies. For example, the Enceladus Flagship PMCS made extensive use of the knowledge gained in developing Europa Lander.

Ocean Worlds in New Frontiers 4. The surprising inclusion of Saturn's ocean-bearing moons Titan and Enceladus in New Frontiers 4 came in part also as a rapid response to Rep. Culberson's successful efforts to mandate in federal law that NASA create an "Ocean Worlds Exploration Program", which facilitated NASA's ability to respond to the major discoveries on the habitability of these moons since V\&V. The general excitement for the Dragonfly winning concept, the submission of design center-vetted biosignature search missions to Enceladus, and the resulting support for the continued inclusion of ocean worlds in the New Frontiers 5 target 
list illustrate the willingness of at least part of the community and NASA leadership to deviate from execution priorities set in the decadal for scientifically compelling reasons. This support is in large part due to new discoveries made at these moons by the Cassini spacecraft in the past decade, and as such this example best illustrates the dilemma of being responsive to such discoveries while staying the Decadal course.

Planetary defense. There has arguably been lesser scientific support for these missions owing to the fact that these missions are not primarily driven by science, despite congressional mandates to track potentially hazardous bodies. The Asteroid Redirect Mission was cancelled after much debate over its value given the multi-billion dollar price tag. Alternate approaches for addressing questions related to planetary defense at fractions of the cost of $A R M$ are currently in development under the new Planetary Defense Coordination Office. The Double Asteroid Redirection Test (DART) was selected as a technology demonstration mission ( $\$ 70$ million). The Near-Earth Object (NEO) Surveillance Mission, though originally proposed as a science mission in Discovery, was selected as an "operational" mission to meet congressional mandates. Both missions will ultimately do science.

Renewed emphasis on Moon science spurred by the Artemis program. The politically mandated Artemis effort to resume human exploration of the Moon has been accompanied by a shift in planetary science funding toward lunar science in the past two years. There are scientific motives (discussed in LEAG white papers) for going back to the Moon, the target of multiple Discovery proposals and a New Frontiers 5 destination in V\&V. The emergence of commercial access to the Moon and the prospect of a human return both provide extra opportunities to fly scientific payloads for which little planning was done in Vision \& Voyages.

\section{Pros and Cons of shifting priorities}

Priorities have shifted at a faster pace than decadal for three reasons: budgetary, political, and scientific (or technological). Arguably, V\&V carefully planned for the first possibility (with decision rules), did not consider the second which turned out to be responsible for the four cases of Section 1, and foresaw but did not attempt to rule on the third (midterm review).

Although budgetary and political changes likely carry the most weight, at least in the short term, the planetary science community may best be able to weigh in on the question of scientifically or technologically driven deviations from Decadal priorities. What if Pluto turns out to teach us about climate change better than Venus? If nuclear fission propulsion cuts travel time to the ice giants by half? If electronics emerge that can withstand Venus surface conditions for years? If an interstellar object is discovered 5 years ahead of crossing Earth's orbit? In the past decade, NASA has called on the National Academies' Committee on Astrobiology and Planetary Sciences (CAPS) for such answers. For example, at NASA's request CAPS deliberated in March 2020 whether to retain the Moon, Trojan asteroids, or Titan as possible destinations of the New Frontiers 5 competition in light of recently funded opportunities to study these locations.

In this Decadal Survey, it may be worth discussing (and perhaps guiding) how to balance the known unknown of upcoming scientific and technical breakthroughs while retaining direction from the scientific consensus couched in it. The table below provides non-exhaustive lists of pros for each side of the balance. 


\begin{tabular}{|c|c|}
\hline Pros - allow changes from Decadal mission priorities & Pros - retain direction from Decadal consensus \\
\hline $\begin{array}{l}\text { - Leverage momentum to grow planetary budget } \\
\text { - Be nimble and agile to time-sensitive opportunities } \\
\text { - Allow unconventional but brilliant concepts to float to the top }\end{array}$ & $\begin{array}{l}\text { - Give each target its turn } \\
\text { - Provide more time to mature mission concepts } \\
\text { - Still allow unconventional, creative ideas to bubble up in } \\
\text { competitive mission classes } \\
\text { - Focus large, sustained investments in technology and } \\
\text { mission studies } \\
\text { - Establish Decadal Survey as credible scientific and political } \\
\text { reference, which ensures political buy-in and financial support }\end{array}$ \\
\hline
\end{tabular}

\section{Options for responsibly enabling responsiveness to discovery}

How might the Decadal Survey achieve a balance that combines as many of the Pros of the table above as possible? Below, we list possible ideas and briefly discuss their intent and potential effects. These ideas are starting points for discussion, not recommendations. They are listed in order of decreasing ease of implementation and increasing amount of Pros from the table.

Open the New Frontiers program to all targets. This straightforward decision combines the Pros of the left column and the last Pro of the right column. Currently, the Discovery program effectively serves this purpose for inner solar system targets, but its cost cap curbs flexibility in formulating missions responsive to discoveries for the outer solar system. Science at inner solar system destinations too may benefit from options afforded under the New Frontiers cost cap.

Allow missions of opportunity alongside large missions. This preserves the Pros of the right column, plus allows some degree of nimbleness and openness to original ideas. So far, aside from instrument contributions to international missions, ride-along spacecraft have only been technology demonstrations (MarCO with InSight, Ingenuity with Perseverance). Cost growth risk has otherwise generally prevented science-driven implementation (e.g., for potential small spacecraft complementing Europa Clipper).

Set aside resources for unforeseen advances warranting priority shifts. This is perhaps best illustrated by the selection of the Comet Interceptor mission for flight by the European Space Agency, a spacecraft whose destination is to be decided after development and launch. In that case, educated guesses can be made on the likelihood of encountering an exciting target (e.g., interstellar object) that ensure the resources are put to good use. More broadly, a planetary discretionary fund could be set at some low percentage of the planetary budget (e.g., equivalent to one Discovery mission per decade) and spent on any unforeseen activity from Research \& Analysis to a small mission or an element of a large mission deemed of equal or higher science return than Decadal priorities.

Edict clear, careful, and comprehensive decision rules. The decision rules of Vision \& Voyages were helpful and clear but they were not always followed. For example, Vision \& Voyages prioritized smaller missions over larger ones, yet two Flagships were prioritized early in the decade just as Discovery and New Frontiers Announcements of Opportunity, expected respectively at a 2 and 5-year cadence, were delayed respectively by about 3 and 2 years. On the other hand, the guidance to fund Research \& Analysis as a top priority was largely followed. In addition to the decision rules provided in Vision \& Voyages, this upcoming Decadal Survey could consider providing decision rules or otherwise foresee the need to react to discoveries and acknowledge that priorities can change beyond its purview because of political reasons. 